\title{
Genetic testing for hereditary hemorrhagic telangiectasia
}

\author{
Yeltay Rakhmanov ${ }^{1}$, Paolo Enrico Maltese ${ }^{1 \star}$, Stefano Paolacci ${ }^{2}$, Carla Marinelli ${ }^{1}$, Raul Ettore Mattassi ${ }^{3}$, \\ Bruno Amato ${ }^{4}$, Tommaso Beccari ${ }^{5}$, Munis Dundar ${ }^{6}$ and Matteo Bertelli ${ }^{1,2}$
}

\begin{abstract}
Hereditary hemorrhagic telangiectasia (HHT) is an autosomal dominant vascular dysplasia characterized by telangiectases and arteriovenous malformations. These lesions cause bleeding, particularly in the nose, gastrointestinal tract and brain. HHT has incomplete penetrance, variable expressivity and genetic heterogeneity. De novo mutations associated with the onset of sporadic HHT have been reported. This Utility Gene Test was prepared on the basis of an analysis of the literature and existing diagnostic protocols. It is useful for confirming diagnosis, as well as for differential diagnosis, couple risk assessment and access to clinical trials.
\end{abstract}

Keywords: Hereditary hemorrhagic telangiectasia, ACVRL1, ENG, GDF2, SMAD4, EBTNA UTILITY GENE TEST

${ }^{1}$ MAGI's Lab, Rovereto, Italy

${ }^{2}$ MAGI Euregio, Bolzano, Italy

${ }^{3}$ Center for Vascular Malformations, "Stefan Belov", Clinical Institute Humanitas "Mater

Domini", Castellanza (Varese), Italy

${ }^{4}$ Department of Clinical Medicine and Surgery at University of Naples Federico II, Naples, Italy

${ }^{5}$ Department of Pharmaceutical Sciences, University of Perugia, Perugia, Italy

${ }^{6}$ Department of Medical Genetics, Erciyes University Medical School, Kayseri, Turkey

${ }^{*}$ Corresponding author: P. E. Maltese E-mail: paolo.maltese@assomagi.org DOI: 10.2478/ebtj-2018-0031
(C) 2018 Authors. This work was licensed under the Creative Commons AttributionNonCommercial-NoDerivs 3.0 License.

\section{Hereditary hemorrhagic telangiectasia}

(Other synonyms: Telangiectasia, hereditary hemorrhagic, type 1; telangiectasia, hereditary hemorrhagic of Rendu, Osler and Weber; telangiectasia, hereditary hemorrhagic, type 2; juvenile polyposis/hereditary hemorrhagic telangiectasia syndrome)

\section{General information about the disease}

Hereditary haemorrhagic telangiectasia (HHT) can be subdivided in hereditary hemorrhagic telangiectasia, type 1 (OMIM disease 187300), and type 2 (OMIM disease 600376), juvenile polyposis/hereditary hemorrhagic telangiectasia syndrome (OMIM disease 175050). All these conditions are characterized by recurrent epistaxis, cutaneous telangiectasia, and visceral arteriovenous malformations (AVMs) that affect the lungs, gastrointestinal tract, liver, brain and other internal organs (1).

The population prevalence rate varies according to different authors. Porteus et al. (2) documented a minimum prevalence of 1 in 40000 . Bideau et al. (3) reported a higher prevalence of HHT cases (1/2300) in an isolated French valley. HHT has autosomal dominant inheritance with incomplete penetrance, and sporadic cases due to de novo mutation have also been described (4). By the age of 20 years, $50 \%$ of individuals have developed some signs of HHT (5).

HHT appears with nosebleeds, and telangiectases on the lips, hands and oral mucosa. Telangiectases in the nasal and gastrointestinal mucosa, and brain arteriovenous malformations generally present with bleeding (6).

Clinical diagnosis of HHT is established when three out of four Curaçao's diagnostic criteria are met. These criteria are: 1) recurrent spontaneous mild to severe epistaxis; 2) multiple mucocutaneous telangiectases; 3) AVMs or telangiectases in internal organs, such as lungs, brain, liver, intestines, stomach and spinal cord; 4) more than one affected family member (7). 
The differential diagnosis should consider von Willebrand disease, ataxia-telangiectasia, calcinosis, Raynaud phenomenon, sclerodactyly, telangiectasia syndrome, capillary malformation-arteriovenous malformation and hereditary benign telangiectasia.

Identification of a heterozygous pathogenic variant in ACVRL1 (OMIM gene 601284), ENG (OMIM gene 131195), GDF2 (OMIM gene 605120), or SMAD4 (OMIM gene 600993) establishes the diagnosis (8).

All these genes are involved in the transforming growth factor beta/bone morphogenetic protein (TGF-beta/BMP) pathway. ACVRL1 encodes a type I cell-surface receptor for the TGF-beta superfamily of ligands, ENG encodes the glycoprotein most expressed in the vascular endothelium and is necessary for the assembly of the TGF-beta receptor complex, GDF2 encodes a secreted ligand of the TGF-beta superfamily of proteins, SMAD4 encodes a member of the Smad family of signal transduction proteins. In response to TGF-beta signalling, small proteins are activated by serine/ threonine receptor kinases through phosphorylation (9).

Pathogenic variants may include missense, nonsense, splicing, small insertions, small deletions, small indels, gross deletions, duplications.

\section{Aims of the test}

- To determine the gene defect responsible for the disease;

- To confirm clinical diagnosis;

- To assess the recurrence risk and perform genetic counselling for at-risk/affected individuals.

\section{Test characteristics}

\section{Specialist centers/ Published Guidelines}

The test is listed in the Orphanet database and is offered by 24 accredited medical genetic laboratories in the EU, and in the GTR database, offered by 18 accredited medical genetic laboratories in the US.

Guidelines for clinical use of the test are described in $\mathrm{Ge}$ netics Home Reference (ghr.nlm.nih.gov) and Gene Reviews (6).

\section{Test strategy}

A multi-gene next generation sequencing panel is used for the detection of nucleotide variations in coding exons and flanking introns of the above genes. Potentially causative variants and region with low coverage are Sanger-sequenced. Sanger sequencing is also used for family segregation studies.

Multiplex Ligation Probe Amplification (MLPA) is used to detect insertions and deletions in ACVRL1 and ENG .

To perform molecular diagnosis, a single sample of biological material is normally sufficient. This may be $1 \mathrm{ml}$ peripheral blood in a sterile tube with $0.5 \mathrm{ml} \mathrm{K}$ EDTA or $1 \mathrm{ml}$ saliva in a sterile tube with $0.5 \mathrm{ml}$ ethanol $95 \%$. Sampling rarely has to be repeated.

Gene-disease associations and the interpretation of genetic variants are rapidly developing fields. It is therefore possible that the genes mentioned in this note may change as new scientific data is acquired. It is also possible that genetic variants today defined as of "unknown or uncertain significance" may acquire clinical importance.

\section{Genetic test results \\ Positive}

Identification of pathogenic variants in the above genes confirms the clinical diagnosis and is an indication for family studies.

A pathogenic variant is known to be causative for a given genetic disorder based on previous reports, or predicted to be causative based on loss of protein function or expected significant damage to proteins or protein/protein interactions. In this way it is possible to obtain a molecular diagnosis in new/ other subjects, establish the risk of recurrence in family members and plan preventive and/or therapeutic measures.

\section{Inconclusive}

Detection of a variant of unknown or uncertain significance (VUS): a new variation without any evident pathogenic significance or a known variation with insufficient evidence (or with conflicting evidence) to indicate it is likely benign or likely pathogenic for a given genetic disorder. In these cases, it is advisable to extend testing to the patient's relatives to assess variant segregation and clarify its contribution. In some cases, it could be necessary to perform further examinations/tests or to do a clinical reassessment of pathological signs.

\section{Negative}

The absence of variations in the genomic regions investigated does not exclude a clinical diagnosis but suggests the possibility of:

- alterations that cannot be identified by sequencing, such as large rearrangements that cause loss (deletion) or gain (duplication) of extended gene fragments;

- sequence variations in gene regions not investigated by this test, such as regulatory regions (5' and 3' UTR) and deep intronic regions;

- variations in other genes not investigated by the present test.

\section{Unexpected}

Unexpected results may emerge from the test, for example information regarding consanguinity, absence of family correlation or other genetically-based diseases.

\section{Risk for progeny}

In autosomal dominant transmission, the probability that an affected carrier transmit the variant to his/her children is $50 \%$ in any pregnancy, irrespective of the sex of the child conceived.

\section{Limits of the test}

The test is limited by current scientific knowledge regarding the gene and disease. 
Analytical sensitivity (proportion of positive tests when the genotype is truly present) and specificity (proportion of negative tests when the genotype is not present)

NGS Analytical sensitivity $>99.99 \%$, with a minimum coverage of 10X; Analytical specificity $99.99 \%$.

SANGER Analytical sensitivity $>99.99 \%$; Analytical specificity 99.99\%.

MLPA Analytical sensitivity $>99.99 \%$; Analytical specificity 99.99\%.

Clinical sensitivity (proportion of positive tests if the disease is present) and clinical specificity (proportion of negative tests if the disease is not present)

Clinical sensitivity: it is reported that the mutation detection rate for ENG and ACVRL1 ranged from $85 \%$ if the ordering physician specifically reported the patient to have all four Curaçao diagnostic criteria (Epistaxis, Telangiectases, AVM, Family History) (8).

Clinical specificity: Data not available

\section{Prescription appropriateness}

The genetic test is appropriate when:

a) the patient meets the diagnostic criteria for HHT;

b) the sensitivity of the test is greater than or equal to that of tests described in the literature.

\section{Clinical utility}

\begin{tabular}{|l|r|}
\hline Clinical management & Utility \\
\hline Confirmation of clinical diagnosis & Yes \\
\hline Differential diagnosis & Yes \\
\hline Couple risk assessment & Yes \\
\hline
\end{tabular}

Availability of clinical trials can be checked on-line at https://clinicaltrials.gov/

\section{References}

1. Dupuis-Girod S, Giraud S, Decullier E, Lesca G, Cottin V, Faure F, Merrot O, Saurin JC, Cordier JF, Plauchu H. Hemorrhagic hereditary telangiectasia (Rendu-Osler disease) and infectious diseases: an underestimated association. Clin Infect Dis 2007; 44(6): 841-5.

2. Porteus MEM, Burn J, Proctor SJ. Hereditary haemorrhagic telangiectasia, a clinical analysis. J Med Genet 1992; 29(8): 527-30.

3. Bideau A, Brunet G, Heyer E, Plauchu H, Robert JM. An abnormal concentration of cases of Rendu-Osler disease in Valserine valley of the French Jura: a genealogical and demographic study. Ann Hum Biol 1992; 19(3): 233-47.

4. Plauchu $H$, de Chadarévian JP, Bideau A, Robert JM. Age-related clinical profile of hereditary hemorrhagic telangiectasia in an epidemiologically recruited population. Am J Med Genet 1989; 32(3): 291-97.

5. McDonald J, P Bayrak-Toydemir P, Pyeritz RE. Hereditary hemorrhagic telangiectasia: an overview of diagnosis, management, and pathogenesis. Genet Med 2011; 13(7): 607-16.

6. Gedge F, McDonald J, Phansalkar A, Chou LS, Calderon F, Mao R, Lyon E, Bayrak-Toydemir P. Clinical and analytical sensitivities in hereditary hemorrhagic telangiectasia testing and a report of de novo mutations. J Mol Diagn 2007; 9(2): 258-65.

7. https://curehht.org/understanding-hht/diagnosis-treatment/ diagnostic-criteria-hht/

8. McDonald J, Pyeritz RE. Hereditary Hemorrhagic Telangiectasia. In: MP Adam, HH Ardinger, RA Pagon, SE Wallace, LJH Bean, et al., editors. GeneReviews(R) 1993; Seattle (WA).

9. http://www.genecards.org/

10. J Richards-Yutz, K Grant, EC Chao, SE Walther, A Ganguly. Update on molecular diagnosis of hereditary hemorrhagic telangiectasia. Hum Genet 2010; 128(1): 61-77. 